\title{
RDUS
}

Revue de DROIT

UNIVERSITÉ DE SHERBROOKE

Titre : $\quad$ LES RAPPORTS DE FORCE ENTRE LES MAJORITÉS ET LES MINORITÉS DE LANGUE OFFICIELLE AU CANADA

Auteur(s): $\quad$ Benoît PELLETIER

Revue : $\quad$ RDUS, 1993-1994, volume 24, numéro 2

Pages: $\quad 255-294$

ISSN : $\quad 0317-9656$

Éditeur : $\quad$ Université de Sherbrooke. Faculté de droit.

URI : $\quad$ http://hdl.handle.net/11143/13370

DOI : https://doi.org/10.17118/11143/13370 
Page vide laissée intentionnellement. 


\title{
ARTICLE
}

\section{LES RAPPORTS DE FORCE ENTRE LES MAJORITÉS ET LES MINORITÉS DE LANGUE OFFICIELLE AU CANADA}

\begin{abstract}
par Benoît PELLETIER*
Le caractère distinct du Québec est issu de l'évolution triplement séculaire d'un peuple, d'une nation qui constitue aujourd'hui le foyer le plus viable et le plus important de la langue et de la culture françaises au Canada. Comme on s'en doute, la préoccupation constante des Québécois francophones pour la survie et l'épanouissement de la langue française en Amérique a forgé au cours des ans les relations qu'ils ont entretenues avec la minorité anglophone du Québec ainsi qu'avec leurs partenaires canadiens. Dans cet esprit, l'auteur se propose d'examiner d'un peu plus près les rapports de force qui existent entre les communautés de langue officielle au Canada, avec comme toile de fond un examen des rapports fondamentaux qui ont cours entre les deux composantes de ce pays «bicéphale» : le Canada anglais et le Canada français.
\end{abstract}

Quebec's distinct character results from the evolution, over a three-century period, of a people, a nation which, today, is the most viable stronghold of the French language and culture in Canada. Over the years, it has remained evident that French-speaking Quebecers' ongoing preoccupation with the survival and blossoming of the French language in America has coloured their relationship with the anglophone minority in Quebec as well as with the English-speaking majority in the rest of Canada. From this perspective, the writer examines the power struggles occurring between the official language communities in light of some of the major confrontations which have taken place between English and French Canada.

*. Professeur à la Faculté de droit de l'Université d'Ottawa. 
SOMMAIRE

PREMIÈRE PARTIE : $\quad$ LE CARACTÈRE DISTINCT DU

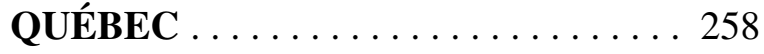

DEUXIÈME PARTIE : $\quad$ LES RAPPORTS FONDAMENTAUX ENTRE LE CANADA ANGLAIS ET LE CANADA FRANÇAIS 269

TROISIÈME PARTIE : $\quad$ LES RAPPORTS ENTRE LA MAJORITÉ FRANÇAISE ET LA MINORITÉ ANGLAISE DU QUÉBEC . . . . . . . . . . . 280

I. La promotion du caractère distinct du Québec et l'essor de sa minorité anglaise . . . . . . . . . . . 282

II. La législation québécoise en matière de langue . . . . . 284

QUATRIÈME PARTIE : LES RAPPORTS ENTRE LES FRANCOPHONES DU QUÉBEC ET CEUX DES AUTRES PROVINCES

CANADIENNES $\ldots \ldots \ldots \ldots \ldots 288$

CONCLUSION ........................... 291 


\section{INTRODUCTION}

La présente étude vise à mieux cerner les rapports de force qui existent entre les majorités et les minorités de langue officielle au Canada. Plus particulièrement, nous tenterons d'identifier les facteurs déterminants de ces mêmes rapports de force, de réfléchir sur la structure d'ensemble des relations linguistiques au Canada, et enfin de cerner les points de convergence et de divergence des intérêts majoritaires et minoritaires en matière d'usage des langues officielles au pays.

Inutile de mentionner que les rapports de force, quels qu'ils soient, sont faits non seulement d'oppositions et d'obstacles mais aussi d'interactions et d'interdépendance. Voilà pourquoi nous mettrons tout autant de soins à mesurer l'ampleur du fossé qui sépare les majorités de langue officielle du Canada des minorités, qu'à tenter d'explorer de nouvelles avenues permettant d'édifier des ponts entre ces groupes linguistiques.

Or, à notre avis, le caractère distinct du Québec est la clé de voûte de toute la dynamique linguistique canadienne; il est le principe qui met en relief et explique les liens réciproques qui existent entre les différentes communautés de langue officielle au pays.

Nous entendons donc faire du caractère distinct du Québec le point de départ de notre étude, la prémisse à partir de laquelle notre analyse découlera, le point de référence ultime. Cette étude de la nature intrinsèque du particularisme québécois constituera d'ailleurs la première partie de notre texte. Nous examinerons par la suite, dans le cadre de la deuxième partie, la structure des rapports généraux qui ont cours entre le Canada anglais et le Canada français, comme il est souvent convenu d'appeler les deux composantes de la dualité canadienne. Ces rapports, en effet, jettent les bases de toute interrelation entre les majorités et minorités de langue officielle du pays. Puis, dans la troisième partie, nous analyserons plus précisément les rapports qui s'établissent entre la minorité anglophone et la majorité francophone du Québec. Enfin, dans la quatrième partie, nous examinerons les relations qui existent entre cette dernière et les minorités francophones des autres provinces du Canada. 
Qu'il nous soit toutefois permis de souligner que nous ne nous attarderons pas à discuter du sort des langues autochtones, ni de la place qui leur revient dans l'ordonnancement linguistique canadien. Rappelons simplement, à cet égard, l'importance qu'il y a à ce que soit formellement reconnu le droit qu'ont les autochtones du Canada, en tant que premiers habitants du pays, non seulement de maintenir mais aussi de développer leurs langues et cultures d'origine.

Aussi est-ce fort de ces dernières remarques que nous entamons dès maintenant l'examen de ce principe fondamental pour le Canada qu'est celui du caractère distinct du Québec.

\section{PREMIÈRE PARTIE : $\quad$ LE CARACTÈRE DISTINCT DU QUÉBEC}

Le Québec s'enorgueillit depuis un certain nombre d'années, avec raison d'ailleurs, de former une société distincte au sein du fédéralisme canadien. De nombreux rapports d'enquête sur l'avenir du pays et propositions constitutionnelles ont d'ailleurs fait écho, de différentes façons, à cette réalité sociologique et historique qu'est l'identité particulière du Québec.

C'est ainsi par exemple que, dans le Rapport Tremblay de 1965, il était énoncé ce qui suit :

«1. La fin première du fédéralisme canadien est de permettre aux deux grandes communautés culturelles dont la population est composée, a) de vivre et de se développer selon leur particularisme respectif; b) de collaborer à l'édification et au progrès d'une patrie commune;

2. la province de Québec assume seule, à l'égard de la culture canadienne-française, les responsabilités que les autres provinces assument en commun à l'égard de la culture anglo-canadienne; $[\ldots]{ }^{1}$.

1. Voir : Rapport de la Commission royale d'enquête sur les problèmes constitutionnels (Rapport Tremblay), Québec, Éditeur officiel, 1956, volume III, tome 2, p. 299. 
En fait, pour les commissaires qui ont participé au Rapport Tremblay, il était clair que le Québec ne constituait pas une province comme les autres².

Dans la même veine, le Rapport Laurendeau-Dunton de 1967 devait reconnaître le rôle du Québec comme défenseur de la communauté française, sur son territoire certes mais aussi dans les autres régions francophones du pays. À l'instar du Rapport Tremblay, il était reconnu par ailleurs que le Québec était différent des autres provinces canadiennes ${ }^{3}$.

En 1967 également, un rapport du Parti libéral du Québec, rédigé sous la direction de Paul Gérin-Lajoie et intitulé «Québec, une société distincte», devait s'inspirer largement de la thèse des deux peuples fondateurs pour interpréter la dynamique politique canadienne, et mettait l'accent sur le concept de statut particulier pour le Québec dans une fédération renouvelée ${ }^{4}$.

2. Voir : «Rapport Tremblay», id., volume I, pp. 65-66.

3. Voir : Rapport de la Commission royale d'enquête sur le bilinguisme et le biculturalisme (Rapport Laurendeau-Dunton), Ottawa, Imprimeur de la Reine, 1967, volume I, p. XXXVIII. Voir également, pp. XXIII et XXIV : «Les deux cultures dominantes, nous l'avons déjà noté, s'incarnent au Canada dans des sociétés distinctes. [...] Et nous avons reconnu dans le Québec les principaux éléments d'une société québécoise distincte. Ainsi en est-il pour l'autre culture dans les provinces anglophones, et, dans une certaine mesure dans le Québec, où le groupe de langue anglaise compense les inconvénients de son statut de minorité par son appartenance à l'ensemble de la société anglophone et par une situation socio-économique très favorable.»

Notons d'ailleurs que la Commission Laurendeau-Dunton avait été constituée en 1963 afin de «faire enquête et rapport sur l'état présent du bilinguisme et du biculturalisme au Canada et recommander les mesures à prendre pour que la Confédération canadienne se développe d'après le principe de l'égalité entre les deux peuples qui l'ont fondée, compte tenu de l'apport des autres groupes ethniques à l'enrichissement culturel du Canada, ainsi que les mesures à prendre pour sauvegarder cet apport [...]» : voir J.-L. GAGNON, Les apostasies, Tome III -- Les palais de glace, Montréal, Les éditions La Presse, 1990, p. 172.

La Commission Laurendeau-Dunton avait également soumis un rapport préliminaire en 1965 : voir Rapport préliminaire de la Commission royale d'enquête sur le bilinguisme et le biculturalisme, Ottawa, Imprimeur de la Reine, 1965, 217 p.

Enfin, les travaux de la Commission prirent fin en 1971, soit trois années après le décès de son co-président et principal inspirateur, Monsieur André Laurendeau.

4. Voir le rapport du Comité des affaires constitutionnelles de la Commission politique de la Fédération libérale du Québec, Québec, une société distincte, Congrès annuel de la Fédération libérale du Québec, le 9 octobre 1967. 
En 1968, René Lévesque et quelques-uns de ses sympathisants rédigèrent Option Québec ${ }^{5}$, un document s'inspirant des travaux des commissions Tremblay et Laurendeau-Dunton. De la première, Lévesque avait retenu l'importance de développer la personnalité collective du Québec, alors que de la seconde, il avait retenu l'idée d'entretenir des discussions «d'égal à égal» entre les «deux majorités» composant le Canada. Plus précisément, Option Québec situait résolument les rapports Québec-Canada au niveau des relations entre États souverains.

Dans le Rapport Pepin-Robarts de 1979 également, les commissaires ont cru bon de proposer la rédaction d'une nouvelle Constitution, dont le préambule confirmerait la reconnaissance historique des anglophones et des francophones, la contribution des autochtones à l'édification du pays, l'apport des autres communautés culturelles à la vitalité nationale, la diversité des régions et la spécificité du Québec. Les commissaires reconnurent au surplus que le gouvernement du Québec se devait d'assumer la responsabilité première pour le maintien et la promotion du patrimoine français sur le territoire québécois ${ }^{6}$. Enfin, les commissaires constatèrent que :

«[L]e Québec est différent et devrait détenir les pouvoirs nécessaires à la préservation et au développement de son caractère distinct au sein d'un Canada viable. Toute solution politique qui ne répondrait pas à cette attente signifierait l'éclatement du Canada»?

5. R. LÉVESQUE, Option Québec, Montréal, Les éditions de l'Homme, 1968, 173 p. Cet ouvrage a fait l'objet d'une nouvelle édition en 1988 : R. LÉVESQUE, Option Québec (texte précédé d'un essai d'André Bernard), Montréal, Les éditions de l'Homme, 1988, 252 p. Il y a lieu de noter qu'une première version d'Option Québec avait été publiée en 1967 : voir Le Devoir, 19 septembre 1967 (p. 5), 20 septembre 1967 (p. 5), et 21 septembre 1967 (pp. 5-6). Il n'en demeure toutefois pas moins que c'est bel et bien en 1968 que le projet politique de Lévesque a pris sa forme définitive.

6. Voir : La Commission de l'unité canadienne, Se retrouver : Observations et Recommandations (Rapport Pepin-Robarts), Ottawa, Éditeur officiel, 1979, volume I, p. 134.

7. $\quad I d .$, p. 92.

Il est intéressant de noter que deux principes fondamentaux ont été spécifiquement reconnus par les commissaires : la dualité et le régionalisme. Ces deux caractéristiques constituaient selon eux les principaux piliers de l'ensemble politique canadien.

Il y a lieu de noter par ailleurs que les commissaires ont identifié -- dans id., p. 24 -- six éléments qui sont autant de caractères originaux de la société québécoise : histoire, langue, 
Publiée dans le contexte référendaire de 1980, La nouvelle entente Québec-Canada : proposition du gouvernement du Québec pour une entente d'égal à égal : la souveraineté-association, privilégiait la formation, sinon la reconnaissance de deux communautés, soit le Canada composé de neuf provinces, et le Québec dorénavant constitué en État distinct et libre d'adhérer à une nouvelle union économique avec son voisin canadien.

Plus particulièrement, la «souveraineté-association» était présentée comme étant la garantie idéale dont pourraient bénéficier les Québécois pour assurer leur existence et leur épanouissement en tant que nation distincte, parce qu'elle respecterait à la fois, du moins prétendait-on, le désir d'autonomie du Québec et la nécessité d'entretenir des liens économiques étroits avec ses partenaires canadiens.

Puis vinrent les démarches entourant le «rapatriement» de la Constitution canadienne et, bien entendu, la vive réaction du Québec au compromis de novembre 1981.

C'est ainsi que, le $1^{\text {er }}$ décembre 1981, l'Assemblée nationale du Québec devait adopter une résolution dans laquelle elle déclarait ne pouvoir accepter le projet de «rapatriement» de la Constitution du Canada, à moins qu'un certain nombre de conditions ne soient rencontrées. La résolution en question était libellée en partie comme suit :

«L'Assemblée nationale du Québec,

code civil, origine commune, sentiments et politique. Au chapitre de l'origine commune, les commissaires soulignèrent «que le Québec est et demeurera essentiellement français, linguistiquement et ethniquement» : voir p. 24. Au chapitre des sentiments des Québécois, les commissaires mentionnèrent que «[1]a mise en commun de leurs désirs, de leurs aspirations, le partage même de craintes analogues au sein de cette collectivité sont peut-être les témoignages les plus probants du caractère culturel distinctif du Québec» : voir p. 25. Enfin, en ce qui concerne la politique en tant que caractère distinctif du Québec, les commissaires rappelèrent que «depuis quelques années on a vu surgir toute une série d'hommes politiques nouveaux, tous poussant au développement politique et social de leur province et de sa population, s'employant vigoureusement à faire servir les ressources de l'État provincial à la réalisation de ces desseins collectifs» : voir p. 25. 
rappelant le droit du peuple québécois à disposer de lui-même, et exerçant son droit historique à être partie prenante et à consentir à tout changement dans la constitution du Canada qui pourrait affecter les droits et les pouvoirs du Québec.

déclare qu'elle ne peut accepter le projet de rapatriement de la constitution sauf si celui-ci rencontre les conditions suivantes:

1. on devra reconnaître que les deux peuples qui ont fondé le Canada sont foncièrement égaux et que le Québec forme à l'intérieur de l'ensemble fédéral canadien une société distincte par la langue, la culture, les institutions et qui possède tous les attributs d'une communauté nationale distincte; $[. ..] \gg^{8}$.

Un peu plus tard, soit le 19 décembre 1981, dans une lettre adressée à la première ministre de Grande-Bretagne, Mme Margaret Thatcher, le premier ministre Lévesque devait évoquer le fait que le Québec...

«qui a des traditions juridiques, religieuses et historiques différentes du reste du Canada et qui vit dans un contexte social, politique et économique qui lui est propre, établit nécessairement dans ses lois des distinctions très légitimes qui ont pour but de protéger son intégrité en tant que société distincte, entourée d'une culture anglophone dominant tout le continent nord-américain» ${ }^{9}$.

Dans la même lettre, René Lévesque devait ajouter :

«À notre avis, l'opposition du Gouvernement du Québec à l'Adresse canadienne est appuyée par la majorité des Québécois pour qui, et ce sans égard à leur allégeance politique, la situation actuelle constitue une crise des plus sérieuses qui touche au coeur même de notre existence comme société distincte» ${ }^{10}$.

8. Pour le texte de la résolution en cause, voir : «Documents relatifs à la Loi constitutionnelle de 1982», (1985) 30 McGill Law Journal 645, pp. 720-721.

9. $\quad$ Voir : id., p. 716.

10. Voir : id., p. 717. 
Le 16 avril 1982, soit la veille de la proclamation de la Loi constitutionnelle de $1982^{11}$, le premier ministre Lévesque émit un communiqué de presse dans le but d'exprimer la réaction du Québec. Plus particulièrement, dans ce message, M. Lévesque insista sur le fait que la Constitution rapatriée ne reconnaissait aucunement, de façon tangible, le caractère et les besoins spécifiques du Québec, société nationale distincte. M. Lévesque fit par ailleurs valoir que la Constitution ignorait le principe même de la dualité canadienne et faisait du Québec une province comme les autres ${ }^{12}$.

En 1985, le Rapport Macdonald devait lui aussi reconnaître le caractère distinct du Québec. Selon les commissaires toutefois, ce caractère devait s'inscrire dans le tout canadien et n'existait que pour autant que l'on puisse le concevoir en relation avec l'expérience canadienne. Les commissaires déclarèrent en outre qu'il était impératif que le caractère distinctif du Québec soit inscrit dans le préambule de la Constitution, mais définissaient cependant celui-ci en des termes strictement linguistiques. C'est ainsi par exemple que les commissaires devaient affirmer :

«L'essence même de la société québécoise découle du fait qu'elle est le principal, sinon l'unique, foyer des dimensions politiques de la vie française au Canada» ${ }^{13}$.

11. Loi constitutionnelle de 1982, annexe B de la Loi de 1982 sur le Canada, 1982, R.-U., ch. 11; L.R.C. (1985), App. II, no 44. La Loi constitutionnelle de 1982 sera ci-après désignée par le terme «Loi de 1982».

12. Pour le texte de ce communiqué de presse, voir : «Documents relatifs à la Loi constitutionnelle de 1982», loc. cit., note 8, pp. 728-730.

Notons d'ailleurs que, dans une lettre qu'il devait adresser au premier ministre Trudeau en date du 17 décembre 1982, M. Lévesque devait réitérer l'idée voulant que le Québec constitue la pierre d'assise des francophones d'Amérique du Nord. Pour le texte de cette lettre, voir : id., pp. 732-735.

13. Voir : Rapport de la Commission royale sur l'union économique et les perspectives de développement du Canada (Rapport Macdonald), Ottawa, Éditeur officiel, 1985, volume III, p. 545.

Notons toutefois qu'à la page 383 du rapport en cause, la formulation est quelque peu différente : «Reconnaissant le caractère distinct de la société québécoise, foyer principal mais non exclusif des francophones au Canada, et acceptant comme une donnée essentielle de la fédération canadienne son caractère dualiste». 
En 1985 également, le gouvernement Lévesque devait soumettre un Projet d'accord constitutionnel ${ }^{14}$, comportant un ensemble de propositions dont l'acceptation par le reste du Canada rendrait possible l'adhésion politique du Québec à la Loi de 1982. Parmi ces conditions, figurait celle ayant trait à la reconnaissance explicite, dans la Constitution du Canada, de l'existence du peuple québécois.

Un mois plus tard, c'était au tour du Parti libéral du Québec de définir lui-aussi les conditions d'adhésion de la province à la Constitution canadienne ${ }^{15}$. Parmi ces conditions figurait l'inscription, dans le préambule d'une nouvelle Constitution, d'un énoncé reconnaissant explicitement le Québec comme foyer d'une société distincte et pierre d'assise de l'élément francophone de la dualité canadienne.

On sait par ailleurs que ce sont ces conditions posées par le Parti libéral du Québec qui donnèrent lieu à la négociation puis à la conclusion de l'Accord du lac Meech en $1987^{16}$. Cet Accord fut complété par une entente constitutionnelle intervenue le 9 juin 1990, à Ottawa ${ }^{17}$.

L'Accord du lac Meech préconisait la reconnaissance du caractère distinct du Québec au sein du Canada, en plus de celle de la dualité canadienne,

14. Projet d'accord constitutionnel: Propositions du gouvernement du Québec, mai 1985. Pour le texte de ce Projet d'accord, voir : A.-G. GAGNON et D. LATOUCHE, Allaire, Bélanger, Campeau et les autres : Les Québécois s'interrogent sur leur avenir, Montréal, Éditions Québec \Amérique, 1991, pp. 116-118.

15. Pour le texte de ces conditions, voir : A.-G. GAGNON et D. LATOUCHE, id., pp. 119-120. Notons d'ailleurs que ces conditions furent reprises par l'ex-ministre délégué aux Affaires intergouvernementales canadiennes, M. Gil Rémillard, à l'occasion d'une allocution prononcée dans le cadre du colloque intitulé «Une collaboration renouvelée du Québec et de ses partenaires dans la Confédération», Mont-Gabriel, 9 mai 1986.

16. Pour le texte de l'Accord du lac Meech, tel qu'intervenu en 1987, voir A.-G. GAGNON et D. LATOUCHE, id., pp. 121-124 (entente politique d'avril 1987, conclue au lac Meech), et pp. 125-136 (textes juridiques endossés par les onze premiers ministres du pays en juin 1987, à l'édifice Langevin (Ottawa)).

17. Cette entente avait été formalisée dans trois documents dont on peut retrouver la teneur dans id., pp. 137-146. 
posée en termes de concentrations géographiques et axée sur des aspects essentiellement linguistiques ${ }^{18}$.

Nous savons tous cependant que l'ultime tentative de juin 1990, visant à sauver l'Accord du lac Meech du «naufrage», n'aboutit pas au résultat escompté. Le dossier constitutionnel fut toutefois ouvert à nouveau un an plus tard, et donna lieu, tour à tour, aux propositions fédérales de septembre $1991^{19}$, au Rapport du comité Beaudoin-Dobbie de février $1992^{20}$ et, enfin, à l'Entente de Charlottetown d'août $1992^{21}$.

18. Il n'est pas inutile de rappeler ici les dispositions pertinentes de l'Accord du lac Meech : «1. La Loi constitutionnelle de 1867 est modifiée par insertion, après l'article 1, de ce qui suit :

\section{RÈGLE INTERPRÉTATIVE}

2.(1) Toute interprétation de la Constitution du Canada doit concorder avec :

a) la reconnaissance de ce que l'existence de Canadiens d'expression française, concentrés au Québec mais présents aussi dans le reste du pays, et de Canadiens d'expression anglaise, concentrés dans le reste du pays mais aussi présents au Québec, constitue une caractéristique fondamentale du Canada;

b) la reconnaissance de ce que le Québec forme au sein du Canada une société distincte.»

Notons par ailleurs que cette clause de l'Accord du lac Meech avait fait l'objet d'un avis juridique de la part de six constitutionnalistes du pays, lequel figurait en annexe à l'entente constitutionnelle du 9 juin 1990 : voir A.-G. GAGNON et D. LATOUCHE, id., pp. 141-142.

19. Voir : Bâtir ensemble l'avenir du Canada - propositions, Ottawa, Ministère des Approvisionnements et Services Canada, 1991, 60 p.

20. Voir : Rapport du Comité mixte spécial sur le renouvellement du Canada (Rapport Beaudoin-Dobbie), Un Canada renouvelé, Ottawa, Imprimeur de la Reine, 1992, 125 p.

21. Voir : Rapport du consensus sur la Constitution : texte définitif, Ottawa, Imprimeur de la Reine, 1992, 21 p. Notons qu'un projet de texte juridique fut soumis à la population du pays pendant le référendum portant sur l'Entente de Charlottetown. Ce texte portait la date du 9 octobre 1992.

Il n'est pas inutile de rappeler également que l'Entente de Charlottetown contenait une Clause Canada, destinée à s'incorporer à la Constitution du Canada en tant qu'article 2 de la Loi constitutionnelle de 1867 : 30 \& 31 Vict., R.-U., ch. 3; L.R.C. (1985), App. II, no 27. La Clause Canada en question comportait notamment les dispositions suivantes : «2. (1) Toute interprétation de la Constitution du Canada, notamment de la Charte canadienne des droits et libertés, doit concorder avec les caractéristiques fondamentales suivantes :

$[\ldots]$

c) le fait que le Québec forme au sein du Canada une société distincte, 
L'Entente de Charlottetown a connu, comme on le sait, le même sort que l'Accord du lac Meech. On sait par ailleurs qu'aucun événement d'importance n'est intervenu depuis lors dans le dossier constitutionnel canadien, si ce n'est bien entendu l'adoption de la «Modification constitutionnelle de 1993 (NouveauBrunswick) $\gg^{22}$, par laquelle ont été enchâssés dans la Constitution du pays le principe de l'égalité du statut, des droits et des privilèges des communautés de langue officielle du Nouveau-Brunswick, ainsi que celui voulant que la législature et le gouvernement de la province aient le rôle de protéger et de promouvoir cette même égalité.

Nous sommes donc en mesure de constater, à la lumière de cette brève rétrospective historique, que le caractère distinct du Québec découle des particularités historiques, juridiques, linguistiques et culturelles de cette province ${ }^{23}$.

comprenant notamment une majorité d'expression française, une culture qui est unique et une tradition de droit civil;

d) l'attachement des Canadiens et de leurs gouvernements à l'épanouissement et au développement des communautés minoritaires de langue officielle dans tout le pays;

[...]

(2) La législature et le gouvernement du Québec ont le rôle de protéger et de promouvoir la société distincte

(3) Le présent article ne porte pas atteinte aux pouvoirs, droits ou privilèges du Parlement ou du gouvernement du Canada, des législatures ou des gouvernements des provinces, ou des corps législatifs ou des gouvernements des peuples autochtones du Canada, y compris à leurs pouvoirs, droits ou privilèges en matière de langue. $[\ldots] »$

22. Proclamation de 1993 modifiant la Constitution (Loi sur le Nouveau-Brunswick), Gazette du Canada, Partie II, vol. 127, n 7 , TR193-54.

23. Le particularisme québécois tient essentiellement au fait que le Québec est le phare de la présence française en Amérique, le château-fort du peuple canadien-français, la seule société francophone qui soit suffisamment organisée au Canada pour y assurer le rayonnement de la langue française. Le Québec est également le principal garant de la survie et de la transmission d'une culture unique, puisant à la fois à la tradition française d'où elle tire son origine et à certaines des valeurs propres au continent au sein duquel elle s'exprime. Enfin, le Québec se distingue au surplus des autres provinces canadiennes par son régime de droit privé, d'inspiration française et, en conséquence, codifié.

Le Québec s'est aussi longtemps distingué dans le contexte canadien par la foi catholique, intensément vécue, de son peuple. C'était l'époque où l'on pouvait encore dire que la langue était gardienne de la foi, et la foi gardienne de la langue. Cependant, dès 1960, la remise en 
Mais on constate par ailleurs que le Québec, en tant que principal foyer de la francophonie en Amérique, a la responsabilité particulière d'assurer la préservation et la promotion du patrimoine français, et ce non seulement sur son territoire, mais aussi dans l'ensemble du Canada.

Certes, on peut vouloir soutenir que le caractère distinct du Québec est fondé sur le fait que cette province est la seule au Canada qui soit formée d'une majorité de langue française. Mais il y a plus! En fait, ce qui est au coeur du concept de «société distincte», c'est le fait que le Québec est la seule terre en Amérique où les francophones forment une nation, un peuple avec ses institutions et ses lois. Car, il ne faut pas l'oublier, le caractère distinct du Québec est essentiellement lié à sa capacité de s'autodéterminer et de s'accomplir en tant qu'État sur la scène internationale ${ }^{24}$.

question des valeurs religieuses traditionnelles et l'émergence du nationalisme politique en tant qu'instrument par excellence de la cohésion sociale ont fait en sorte que le Québec s'est de moins en moins distingué des autres provinces canadiennes par la ferveur catholique de son peuple.

Enfin, certains aiment invoquer le caractère distinct du Québec au plan des lois sociales et économiques, comme par exemple la Loi sur les valeurs mobilières, L.R.Q., ch. V-1.1; la Loi sur la caisse de dépôt et de placement du Québec, L.R.Q., ch. C-2; la Loi sur les caisses d'épargne et de crédit, L.R.Q., ch. C-4.1; la Loi sur les caisses d'entraide économique, L.R.Q., ch. C-3; la Loi sur les coopératives, L.R.Q., ch. C-67.2; la Loi sur la qualité de l'environnement, L.R.Q., ch. Q-2; la Loi sur les normes du travail, L.R.Q., ch. N-1.1; la Loi sur la santé et la sécurité du travail, L.R.Q., ch. S-2.1; ou la Loi sur les accidents du travail et les maladies professionnelles, L.R.Q., ch. A-3.001. Ces lois illustrent certes l'originalité de la société québécoise et son avant-gardisme dans une foule de domaines, mais elles ne constituent toutefois pas, à notre avis, les principaux éléments du caractère distinctif du Québec. En effet, très souvent ces lois s'articulent autour de principes de base qui sont en fait nord-américains.

24. Le professeur Jacques Brossard, éminent internationaliste québécois, devait affirmer, dans son ouvrage magistral intitulé L'accession à la souveraineté et le cas du Québec -- Montréal, Les Presses de l'Université de Montréal, 1976, p. 188 --, que la nation canadienne-française constitue un «peuple» au sens de la Charte des Nations unies et qu'elle a le droit de s'autodéterminer, mais que dans les faits, par suite de la concentration de la nation canadienne-française sur le territoire du Québec, ce droit pourrait être exercé par «le peuple québécois», soit au nom de la nation canadienne-française, soit en son propre nom. Comme devait en effet l'affirmer le professeur Brossard dans l'ouvrage précité, p. 190 :

«[I]l ne paraît faire aucun doute, de prime abord, que le peuple québécois a non seulement le droit de disposer de lui-même mais celui de choisir "la création d'un État souverain et indépendant" aussi bien que "la libre association ou intégration avec un État indépendant ou l'acquisition de tout autre statut politique librement décidé par son peuple" [...].» 
Le caractère distinct du Québec rejoint donc le fait que celui-ci forme une communauté nationale dans l'ensemble canadien, un peuple qui possède une identité qui lui est propre et qui a par ailleurs la responsabilité particulière de faire la promotion de celle-ci.

On comprend dès lors jusqu'où il peut être légitime pour le Québec de chercher à accroître, sinon à maintenir les pouvoirs lui permettant de faire valoir son originalité, de préserver l'intégrité française du pays et de développer celleci dans tous ses éléments.

Mais on comprend également que le caractère distinct du Québec, ainsi défini en termes de «foyer national du Canada français», implique en lui-même que le fédéralisme canadien soit perçu comme étant «bi-national» ${ }^{25}$, voire

Le professeur Brossard devait également préciser que le terme «peuple québécois» peut être interprété de façon stricte, c'est-à-dire comme n'incluant que les Québécois qui appartiennent à la nation canadienne-française, ou encore de façon large, incluant cette fois-ci tous les ressortissants du Québec, qu'ils appartiennent ou non à la nation canadienne-française : voir id., p. 180. Chose certaine, devait toutefois ajouter le professeur Brossard, les anglophones du Québec ne remplissent pas à eux seuls les conditions requises pour constituer un «peuple» distinct au sens de la Charte des Nations Unies, faute de pouvoir former un État viable : voir id., p. 181.

25. Le professeur Jacques Brossard devait affirmer, dans id., p. 173 :

«Par contre, lorsqu'on parle des "deux nations" du Canada, il n'est pas certain que l'on puisse mettre en parallèle la nation canadienne-française et la "nation canadienne-anglaise". Les Canadiens anglais paraissent être eux-mêmes très sceptiques quant à l'existence de celle-ci; il ne faut d'ailleurs pas oublier que plus du tiers des anglophones du Canada ne sont pas d'origine britannique. En réalité, pour les anglophones, leur "nation", même au sens sociologique du terme, c'est le Canada.»

Le professeur Brossard préféra donc utiliser l'expression «peuple anglo-canadien», plutôt que «nation canadienne-anglaise», pour désigner l'ensemble des Canadiens d'origine et de langue anglaises.

Jacques Brossard ajouta cependant, p. 174 :

«Il ne fait pourtant aucun doute que le Canada anglais [...] comporte lui aussi, dans son ensemble et malgré ses fortes diversités régionales, tous les éléments d'une nation au sens sociologique du terme $[\ldots]$.»

De même, le professeur Brossard préféra parler du «peuple québécois» plutôt que de la «nation québécoise», puisqu'il lui semblait «difficile de conclure de façon certaine qu'il existe présentement une «nation québécoise» distincte, en tant que nation, de la «nation canadienne-française» dont elle se serait détachée : voir p. 179.

Le professeur Brossard devait toutefois ajouter, quelques lignes plus loin, que «[p]our la plupart, $[\ldots]$ les termes "peuple" et "nation" paraissent être plus ou moins synonymes [...]» : 
«multi-national» en cherchant à la limite à y inclure les peuples autochtones du Canada $^{26}$. Cette «dualité canadienne» suppose elle-même, idéalement, l'égalité de chacune des communautés nationales composant le pays.

Voilà donc quels sont les principes qui posent les jalons des rapports fondamentaux entre le Canada français et le Canada anglais ${ }^{27}$. Ces rapports dépendent en effet essentiellement de la reconnaissance de la dualité nationale du Canada et de la recherche de l'égalité ou de l'équilibre ${ }^{28}$ entre les deux

voir p. 180.

En ce qui nous concerne, nous entendons laisser aux internationalistes eux-mêmes le soin d'apporter ces nuances subtiles entre les termes «peuple» et «nation». Ainsi, plutôt que d'accorder crédit à ces questions de sémantique, nous entendons utiliser indistinctement les deux termes en question dans le présent texte.

26. Le professeur Jacques Brossard a affirmé, dans id., p. 187, «que les "Esquimaux" [Inuits] et Amérindiens du Canada ne constituaient pas "un peuple" au sens de la Charte des Nations unies, compte tenu de leur petit nombre, de leur dispersion territoriale (à travers le Québec comme à travers le Canada) et de leurs subdivisions en "ethnies" diversifiées.»

Il n'en demeure toutefois pas moins que les «autochtones» du Canada constituent une minorité «ethnique» d'un type particulier, puisqu'ils descendent des tout premiers habitants du Canada et que de plus en plus ils revendiquent, avec un appui populaire non négligeable, le statut de «peuple» distinct au sein du Canada. On n'a qu'à penser en effet, à cet égard, à la défunte Entente de Charlottetown qui se proposait notamment de consacrer le droit inhérent à l'autonomie gouvernementale des «peuples autochtones du Canada».

Reste que les autochtones, même s'ils devaient constituer un «peuple» à la limite, n'ont toujours eu qu'une influence très secondaire sur la dynamique linguistique au Canada. Voilà d'ailleurs pourquoi nous ne nous attarderons, dans le présent article, qu'à examiner les rapports de force qui existent les deux grandes communautés de langue officielle du pays : la francophone et l'anglophone.

27. Comme nous l'avons déjà vu (supra, note 25), l'expression «Canada anglais» recouvre en réalité une diversité ethnique. Pour les Québécois cependant, cette expression regroupe tous les parlants anglais, y compris les ethnies immigrantes non britanniques.

28. Comme devait en effet l'affirmer Jean-Louis Gagnon, seul survivant des trois présidents qui ont veillé à l'élaboration du Rapport de la Commission royale d'enquête sur le bilinguisme et le biculturalisme, dans op. cit., note 3, pp. 172-173 :

«Mais tant pour les peuples que pour les individus, le principe de l'égalité ne s'exprime pas toujours par un calcul d'arithmétique. S'il obéit aussi à des lois qui échappent au jeu des nombres, c'est qu'il a pour source cet idéal de justice commun aux nations libres et policées. Dans cette perspective, le principe de l'égalité exige que les droits fondamentaux de tout citoyen canadien, civils ou culturels, soient respectés de tous, de même qu'il signifie que les droits collectifs propres à chacune des deux communautés linguistiques qui donnent au Canada sa dimension biculturelle puissent s'exercer librement.»

Permettons-nous simplement de préciser ces propos de Jean-Louis Gagnon en affirmant que le principe de l'égalité prend souvent forme dans la recherche d'un équilibre raisonnable 
communautés de langue officielle du pays. Proposons-nous, dès maintenant, d'examiner l'ensemble de ces questions de façon un peu plus détaillée.

\section{DEUXIÈME PARTIE : LES RAPPORTS FONDAMENTAUX ENTRE LE CANADA ANGLAIS ET LE CANADA FRANÇAIS}

En 1867, lors de la création du Canada, l'adoption de l'article 133 de la Loi constitutionnelle de $1867^{29}$ devait constituer un compromis historique et politique de la plus haute importance. Comme devait en effet l'affirmer l'un des «pères de la fédération canadienne», John A. MacDonald, lors des grands débats parlementaires de 1865 :

«Je conviens avec l'hon. député d'Hochelaga qu'aujourd'hui cela est laissé à la majorité; mais afin d'y remédier, il a été convenu dans la conférence d'introduire cette disposition dans l'acte impérial. Cela a été proposé par le gouvernement canadien par crainte qu'il survienne plus tard un accident, et les délégués de toutes les provinces ont consenti à ce que l'usage de la langue française formât l'un des principes sur lesquels serait basée la confédération, et que son usage, tel qu'il existe aujourd'hui, fût garanti par l'acte impérial» ${ }^{30}$.

À cela, Georges-Étienne Cartier devait ajouter :

«II]l fallait aussi protéger la minorité anglaise du Bas-Canada, relativement à l'usage de sa langue, parce que dans le parlement local du Bas-Canada la majorité sera composée de Canadiens-Français. Les membres de la conférence ont voulu que cette majorité ne pût pas décréter l'abolition de l'usage de la langue anglaise dans la législature locale du Bas-Canada, pas plus que la majorité anglaise de la législature fédérale ne pourra le faire pour la langue française.

entre partenaires de forces inégales certes, mais néanmoins perçus comme égaux en droit.

29. Loi constitutionnelle de 1867, précitée, note 21. Cette loi sera ci-après désignée par le terme «Loi de 1867».

30. Débats parlementaires sur la question de la confédération des provinces de l'Amérique Britannique du Nord, 3e session, 8e Parlement provincial du Canada, Québec, Imprimeurs parlementaires, 1865, p. 943. 
J'ajouterai aussi que l'usage des deux langues sera garanti dans l'acte impérial basé sur ces résolutions» ${ }^{31}$.

Cartier et MacDonald virent donc en l'article 133 une disposition susceptible d'assurer la parité entre l'usage du français à Ottawa et l'usage de l'anglais à Québec. Cette même conception semblait d'ailleurs être partagée par d'autres «pères de la fédération», dont Galt, Langevin et Dorion ${ }^{32}$.

Plus précisément, l'article 133 était historiquement perçu comme offrant des garanties constituant l'assise même de la fédération canadienne, ou comme reflétant les termes d'une entente fondamentale intervenue entre les «pères de la fédération» relativement à la protection de la minorité anglaise du Québec et de la minorité française du Canada.

La jurisprudence, elle aussi, a de tout temps reconnu que l'article 133 constituait une disposition intrinsèquement liée aux conditions du fédéralisme canadien, voire une condition essentielle à l'adhésion même du Québec au compromis fédératif.

C'est ainsi par exemple que, dans l'affaire Blaikie, la Cour supérieure du Québec avait affirmé, sous la plume du juge en chef Deschênes, que l'adoption de l'article 133 de la Loi de 1867 était fondée sur...

«[...] l'intention des Pères de la Confédération de soustraire la question de l'usage de ces deux langues anglaise et française à la possibilité de l'arbitraire ou du caprice ou même, tout simplement, de la volonté perçue comme légitime d'une majorité, qu'elle fût anglophone dans le Parlement central ou francophone dans la législature de Québec» ${ }^{33}$.

31. Ibid.

32. Voir à cet égard : A.L.C. DE MESTRAL et W. FRAIBERG, «Language Guarantees and the Power to Amend the Canadian Constitution», (1966-67) 12 McGill Law Journal 502, p. 514 (note en bas de page $n^{\circ} 53$ ).

33. Blaikie et autres c. Procureur général du Québec et Procureur général du Canada, [1978] C.S. 37, p. 53. 
Dans la même veine, le juge Bélanger devait affirmer ce qui suit, lorsque l'affaire Blaikie fut soumise à la Cour d'appel du Québec :

«À mon sens, en accordant aux provinces le pouvoir d'amender leur Constitution, le Législateur n'a pas eu l'intention de leur accorder le droit de changer les garanties prévues à l'article 133 [...]. Il est bien sûr que le Législateur n'a pas voulu que les parties à l'état fédératif puissent en modifier unilatéralement les conditions» ${ }^{34}$.

Toujours dans le cadre de cette décision de la Cour d'appel du Québec, le juge Casey avait lui aussi reconnu en ces termes le caractère fondamental de l'article 133 :

«The B.N.A. Act was an attempt to resolve the problems unavoidably caused by the separate existence and divided population of the Colonies. A basic requirement of this solution was that minority rights be respected -- hence sec. 133. It follows that the right to amend conferred on provincial legislatures by sec. 92(1) cannot be extended to cover a provision such as sec. 133. "Constitution of the Province" must be limited to the manner in which provincial legislative authority is exercised: it cannot be read to permit the modification of that authority or the amendment or nullification of a provision that was designed to serve the best interest of all. For these reasons the argument that sec. 133 is severable and that the provincial part of that section forms part of the "Constitution of the Province" is without substance.

Nor I am impressed by the argument that the totality of legislative authority was divided between the central and local governments and that the latter, there being no exclusion in sec. 92(1) comparable to that of sec. 91(1), are empowered to amend sec. 133. Apart from the fact that the basic premise is unfounded, the unalterability of sec. 133 results from the intention of Parliament to unite the Provinces» ${ }^{35}$.

34. Procureur général du Québec c. Blaikie et autres, [1978] C.A. 351, p. 360.

35. Id., p. 353. 
Quant à la Cour suprême du Canada, elle s'est contentée, toujours dans l'affaire Blaikie, de faire sienne les motifs du juge en chef Deschênes, tels que renforcés par ceux de la Cour d'appel du Québec ${ }^{36}$.

La Cour suprême profita toutefois de l'affaire MacDonald pour revenir de façon un peu plus explicite sur l'intention qui avait présidé à l'adoption de l'article 133 de la Loi de 1867. C'est ainsi que le juge Beetz a rappelé que cette disposition avait été le résultat «d'un compromis historique intervenu entre les fondateurs quand ils se sont entendus sur les modalités de l'union fédérale» ${ }^{37}$, alors que la juge Wilson a reconnu que l'intention des autorités constituantes avait été, en adoptant l'article 133, «de protéger chacun des deux groupes linguistiques fondateurs de l'empiétement et finalement de la domination de l'autre» ${ }^{38}$.

On constate donc que l'article 133 s'est trouvé à constitutionnaliser dans une certaine mesure l'égalité des langues française et anglaise au Québec et au fédéral, de façon à apporter des protections efficaces en faveur des groupes minoritaires concernés et à apaiser les craintes que ceux-ci éprouvaient de voir leurs droits être remis en question par la majorité.

Mais on constate également que ce qui caractérise au premier chef l'article 133, c'est le fait qu'il constitue un compromis entre les deux peuples fondateurs du pays, à savoir le Canada anglais et le Canada français.

Plus précisément, nous sommes d'avis que non seulement le concept des deux peuples fondateurs a-t-il été à la base de l'adoption de l'article 133 de la Loi de 1867, mais qu'en plus ce même concept a été en mesure de marquer l'ensemble des rapports linguistiques au Canada.

En effet, c'est le concept des deux peuples fondateurs qui a été la source même de l'adoption de l'article 23 de la Loi de 1870 sur le Manitoba ${ }^{39}$, une

36. Voir Procureur général du Québec c. Blaikie, [1979] 2 R.C.S. 1016 [Blaikie no 1], p. 1027.

37. MacDonald c. Ville de Montréal, [1986] 1 R.C.S. 460, p. 496.

38. $\quad I d .$, p. 538.

39. Loi de 1870 sur le Manitoba, 1870, 33 Vict., ch. 3 (Canada); L.R.C. (1985), App. II, no 8. Cette loi sera ci-après désignée par le terme «Loi de 1870». 
disposition qui découlait de la même motivation que celle qui avait amené quelques années auparavant l'adoption de l'article 133 de la Loi de 1867 : protéger chacun des deux groupes fondateurs contre l'intervention législative de l'autre $^{40}$. Mais c'est également le concept des deux peuples fondateurs qui a été à la base du principe de l'égalité des deux langues officielles au Canada, principe dont on retrouve la reconnaissance au paragraphe 16(1) de la Charte canadienne des droits et libertés ${ }^{41}$ ou encore dans la Loi sur les langues officielles de $1988^{42}$.

Il appert en effet que c'est le caractère bi-national du Canada qui justifie l'affirmation du bilinguisme officiel et qui pose, avant toute stipulation du droit positif, le principe fondamental de l'égalité des langues anglaise et française à la grandeur du pays.

L'égalité des langues officielles au Canada ne peut effectivement se concevoir que dans la mesure où on aborde les droits linguistiques sous l'angle historique et sociologique, sous l'angle d'un rapport de force entre deux réalités distinctes s'exprimant au Canada, ayant chacune le droit d'être traitée de façon égale à l'autre et, bien entendu, devant chacune disposer (du moins théoriquement) des mêmes chances de s'épanouir ${ }^{43}$. En fait, cette égalité

40. Sur le parallèle qui existe entre l'article 23 de la Loi de 1870 et l'article 133 de la Loi de 1867, voir : B. PELLETIER, «La modification des dispositions constitutionnelles relatives à l'usage de l'anglais ou du français», (1990) 21 R.G.D. 223, pp. 261-273. Il en ressort notamment que l'article 23 de la Loi de 1870 était pour les Franco-Manitobains une garantie fondamentale «enchâssée» dans la Constitution du pays, à l'instar de ce qu'était l'article 133 de la Loi de 1867 pour les Anglo-Québécois (dans son aspect québécois) et pour les francophones (dans son aspect fédéral).

41. La Charte canadienne des droits et libertés est la Partie I (articles 1 à 34) de la Loi de 1982. Cette charte sera ci-après désignée par le terme «Charte de 1982».

42. Loi concernant le statut et l'usage des langues officielles du Canada, L.C. 1988, ch. 38.

43. Il est intéressant de noter effectivement que le principe même de l'égalité des langues officielles, lorsqu'il est énoncé de façon générale, est fondé non pas sur l'égalité de tous les citoyens mais bien plutôt sur celle des communautés linguistiques concernées. En effet, non seulement l'égalité des deux langues officielles du Canada s'oppose-t-elle au pluralisme linguistique dans les secteurs qu'elle vise, mais en plus s'oppose-t-elle essentiellement à l'idée que le groupe majoritaire tire profit des droits linguistiques reconnus en proportion de son nombre, c'est-à-dire, justement, tire profit de son statut majoritaire. En d'autres termes, le concept de «l'égalité des langues officielles» ne saurait reposer que sur une conception collective, par opposition à personnelle ou individuelle, des droits linguistiques.

Dans cet esprit, permettons-nous d'ajouter que l'égalité des langues officielles au Canada 
linguistique suppose non seulement la reconnaissance de la dualité canadienne, mais aussi l'équilibre dans les relations entre la communauté française et la communauté anglaise du Canada.

Le problème tient toutefois au fait que le concept des deux peuples fondateurs ne jouit en tant que tel d'aucune reconnaissance formelle dans la Constitution du pays. En fait, non seulement ce principe ne jouit-il d'aucune consécration, mais en plus fut-il battu en brèche par la Loi de $1982^{44}$, une loi à tendance centralisatrice et uniformisatrice ${ }^{45}$.

devrait, pour ne pas être que théorique, faire appel à l'application de mesures spéciales en faveur de celle des communautés linguistiques impliquées qui est sociologiquement «désavantagée» par rapport à l'autre : la communauté francophone. Nous reviendrons toutefois sur ce point un peu plus loin.

44. De nombreux auteurs estiment que le révision constitutionnelle de 1982 et l'échec de l'Accord du lac Meech qui en a intimement découlé ont irrémédiablement détruit le rêve dualiste des Québécois. C'est ainsi que le rêve des deux nations, des deux peuples fondateurs ou des deux sociétés distinctes serait, selon eux, devenu désormais impossible à réaliser. Voir par exemple, en ce sens : G. LAFOREST, Trudeau et la fin d'un rêve canadien, Sillery, Éditions du Septentrion, 1992, p. 13 : «L'échec de l'Accord du lac Meech en juin 1990 confirme l'idée selon laquelle la dualité, pivot de l'interprétation du fédéralisme canadien dans l'historiographie canadienne-française puis québécoise, est devenue impossible. Il est devenu irréaliste d'espérer que la vision dualiste, celle qui conçoit le fédéralisme comme reposant sur une entente entre deux sociétés distinctes, entre deux nations ou deux peuples fondateurs, puisse occuper des espaces de pouvoir significatifs dans les infrastructures constitutionnelles canadiennes. C'est donc bel et bien la fin d'un rêve, avec toute la tristesse que cela évoque.»

Nous sommes également d'avis que la Loi de 1982 s'est trouvée à réserver des funérailles de première classe au principe des deux peuples fondateurs, et qu'il y a tout à parier que celui-ci ne renaîtra pas de ses cendres sur le plan juridique. Il n'en demeure toutefois pas moins que ce même principe continue de soulever un intérêt théorique indéniable, en plus d'être encore, en réalité, au coeur même de la composition sociologique du Canada.

Reste que les chances sont extrêmement minces de voir un jour une réforme constitutionnelle au Canada consacrer le principe des deux peuples fondateurs, puisque ce principe n'a guère plus droit de cité au «Canada anglais» et puisque toute révision constitutionnelle d'envergure requiert sinon le consentement unanime de toutes les assemblées législatives canadiennes (voir l'article 41 de la Loi de 1982), à tout le moins le consentement du fédéral et de sept provinces représentant cinquante pour cent de la population totale des provinces canadiennes (voir les articles 38 et 42 de la Loi de 1982).

45. À titre d'exemple, mentionnons que la Charte de 1982, contenue dans la Loi de 1982, favorise une interprétation uniforme des droits et libertés qui y sont énoncés (voir toutefois infra, note 50), et ne reconnaît ni n'accommode d'aucune façon la spécificité québécoise. La Charte de 1982 encourage au contraire l'émergence de valeurs «nationales» (pan- 
Mais le problème tient également au fait que la Charte de 1982 n'envisage l'égalité des langues officielles au Canada que de façon abstraite, plutôt que de façon sociologique.

En effet, au lieu de reconnaître spécifiquement les besoins particuliers de la langue française au Canada, et plus particulièrement au Québec, la Charte de 1982 se trouve à mettre directement en rapport des groupes dont les pouvoirs réels, les besoins de langue ou les chances de survie sont très différents, des groupes sociologiquement dissemblables. Pourtant, tout le monde connaît l'asymétrie de la situation réelle des Anglo-Québécois et des francophones vivant à l'extérieur du Québec ${ }^{46}$. Tout le monde sait également jusqu'à quel point la survie de la langue française peut être menacée même au Québec, où elle est pourtant la langue de la majorité ${ }^{47}$.

canadiennes) et communes à tous les citoyens et citoyennes du Canada, sans exceptions (ou si peu) et sans sensibilité aux particularités québécoises. Par ailleurs, la Charte de 1982 est interprétée en dernier ressort par la Cour suprême du Canada, un tribunal dont la place et le rôle dans le système judiciaire canadien l'incitent à favoriser une intégration des différentes normes juridiques dans un corpus univoque, ainsi que le nivellement de celles-ci.

Quant aux procédures de modification constitutionnelle contenues dans la Partie V de la Loi de 1982, elles accordent un droit de veto au Parlement fédéral sur toute réforme constitutionnelle, mais n'en accordent cependant pas au Québec. En réalité, ces procédures se trouvent à consacrer l'égalité des provinces entre elles.

Enfin, la Loi de 1982 est à peu près silencieuse sur la question du partage des compétences législatives entre le fédéral et les provinces (hormis quelques dispositions particulières en matière de ressources naturelles), et laisse désormais cette même question à la merci du processus de révision constitutionnelle, dont nous avons déjà mentionné le caractère relativement complexe : voir supra, note 44.

46. Sur le sujet, voir : J. WOEHRLING, «Les droits linguistiques des minorités et le projet de modification de la Constitution du Canada (L'Accord du lac Meech)», dans P. PUPIER et J. WOEHRLING (dir.), Langue et droit : Actes du premier congrès de l'Institut international de droit linguistique comparé, Montréal, Wilson \& Lafleur ltée, 1989, p. 310.

47. Comme devaient en effet l'affirmer les auteurs Henri Brun et Guy Tremblay, dans Droit constitutionnel, 2e édition, Montréal, Éditions Yvon Blais, 1990, p. 766 : «[O]n n'a jamais su reconnaître dans le domaine linguistique les besoins spécifiques du Québec, où se trouve la seule majorité francophone du Canada, majorité qui en même temps concentre la principale minorité du Canada et dont la survie reste problématique. Cette minorité n'est jamais prise en compte : la Loi de 1982, comme la nouvelle Loi fédérale sur les langues officielles, ne protègent que les "minorités" linguistiques qui se trouvent à l'intérieur de chaque province.» 
En n'offrant aucune reconnaissance particulière à la langue française au Canada, la Charte de 1982 se trouve donc à nier la réalité sociologique même de ce pays, ainsi qu'à émasculer les droits qu'elle cherche à conférer. Même le principe de l'égalité des langues officielles du Canada, qui est notamment reconnu au paragraphe 16(1) de la Charte de 1982 et qui recèle, comme nous l'avons $\mathrm{vu}^{48}$, une connotation sociologique indéniable, ne s'est jusqu'à maintenant vu reconnaître par la Cour suprême du Canada qu'une portée déclaratoire, dénudée de contenu réel, purement symbolique ${ }^{49}$. Quant à l'article 1 de la Charte de 1982, bien qu'il recèle le potentiel de favoriser une certaine asymétrie dans l'interprétation et l'application des droits découlant de la charte

48. Voir supra, note 43.

49. Voir par exemple l'arrêt Société des Acadiens c. Association of Parents, [1986] 1 R.C.S. 549, où la Cour a examiné le paragraphe 16(1) de la Charte de 1982 à la lumière du paragraphe 16(3), et a conclu que ces dispositions laissaient aux législateurs, plutôt qu'aux tribunaux, le soin d'améliorer le statut égalitaire du français et de l'anglais au Canada. En d'autres termes, selon la Cour, ces dispositions envisagent une réalité simplement progressive des deux langues officielles et laissent au législateur le soin de la réaliser. Comme devait en effet l'affirmer le juge Beetz, p. $579:$ :Je crois qu'il est exact d'affirmer que l'art. 16 de la Charte contient un principe d'avancement ou de progression vers l'égalité de statut ou d'usage des deux langues officielles. Je considère toutefois qu'il est très significatif que ce principe de progression soit lié au processus législatif mentionné au par. 16(3) où se trouve consacrée la règle énoncée dans l'arrêt Jones c. Procureur général du Nouveau-Brunswick, [1975] 2 R.C.S. 182. Comme le processus législatif est, à la différence du processus judiciaire, un processus politique, il se prête particulièrement bien à l'avancement des droits fondés sur un compromis politique.» 


\section{en question ${ }^{50}$, force nous est d'admettre qu'il s'est avéré jusqu'à maintenant n'être que d'une utilité douteuse en matière de droits linguistiques ${ }^{51}$.}

50. L'article 1 de la Charte de 1982 est susceptible d'autoriser les tribunaux, dans l'interprétation qu'ils font des droits et libertés qui y sont énoncés, à tenir compte par exemple de facteurs sociologiques, philosophiques, historiques et politiques. Évidemment, ce qui est «raisonnable» et ce qui se justifie dans «le cadre d'une société libre et démocratique», pour emprunter à la terminologie de l'article 1 en question, peut varier d'une société à une autre, dépendamment en partie des facteurs susmentionnés. Une certaine asymétrie pourrait donc résulter d'une application des droits et libertés de la Charte de 1982 qui tiendrait compte, dans chaque cas, du contexte social dans lequel ceux-ci s'inscrivent.

Il faut bien admettre cependant que l'article 1 de la Charte de 1982 n'a que très peu favorisé, jusqu'à présent, l'asymétrie en matière d'interprétation judiciaire. Peut-être est-ce là la conséquence directe du test rigide qui avait été élaboré par la Cour suprême elle-même dans l'affaire $R$. c. Oakes, [1986] 1 R.C.S. 103? Mais peut-être est-ce au contraire le fait d'un manque d'imagination de la part des juristes, qui ne sauraient pas faire valoir adéquatement les dispositions de l'article 1 en question? Cette dernière hypothèse est du moins celle qui fut développée par Mme Bertha Wilson, après qu'elle eut quitté la Cour suprême du Canada, dans son article intitulé «Constitutional Advocacy», (1992) 24 Ottawa Law Review 265, p. 273 : «I don't believe that counsel have yet realized the importance of taking a broader approach to Charter litigation although I think that the Court's recent emphasis on the contextual approach must have brought home to them the need to inform themselves thoroughly on the social context in wich the issue arises and that they must appreciate it not only intellectually but emotionally as well. [...] So please don't treat Charter cases as if you were dealing with mechanics' liens. Approach them with a bit of imagination. Make clear to the Court the larger social implications of the issue before it, but make sure that you completely understand those implications yourself.»

51. On n'a qu'à penser ici à l'arrêt Ford c. P. G. (Québec), [1988] 2 R.C.S. 712. Dans cette affaire, en effet, se posait la question de savoir si les articles 58 et 69 de la Loi 101 (voir infra, note 57) étaient inopérants parce que contraires à la Charte des droits et libertés de la personne du Québec (L.R.Q., ch. C-12) ou à la Charte de 1982.

Les articles en cause prévoyaient que l'affichage public, la publicité commerciale et l'usage d'une raison sociale ne pouvaient se faire qu'en français au Québec. Après avoir reconnu que ces dispositions allaient à l'encontre de la liberté d'expression, telle que protégée par les chartes en cause, la Cour suprême du Canada s'est demandée si, néanmoins, ces dispositions n'étaient pas justifiées en vertu de l'article 9.1 de la Charte des droits et libertés de la personne ou de l'article 1 de la Charte de 1982.

Sur ces questions, la Cour estima que la preuve n'avait pas été faite que l'exigence de l'emploi exclusif du français était nécessaire pour atteindre l'objectif législatif qui était visé par la Loi 101, ni que cette même exigence était proportionnée à l'objectif en question : voir Ford, pp. 779-780. En conséquence, la Cour en vint à la conclusion que l'usage exclusif du français ne résistait pas à l'examen fondé sur le critère de proportionnalité, et ne reflétait pas la réalité de la société québécoise : voir Ford, p. 780. La Cour a donc déclaré inopérants les articles 58 et 69 de la Loi 101, parce que ceux-ci étaient contraires aux deux chartes invoquées en l'espèce. 
Nous estimons donc que, plutôt que d'être purement théorique comme c'est le cas actuellement, l'égalité des langues officielles au Canada devrait donner lieu à des mesures concrètes, à des dispositions s'incarnant dans la réalité et visant à réaliser dans les faits l'égalité des deux grandes communautés linguistiques du Canada, ou du moins permettant à celles-ci de tendre vers cette égalité.

La Cour suprême du Canada, en reconnaissant que les droits linguistiques sont fondés sur un compromis politique plutôt que sur des principes, contrairement aux droits fondamentaux traditionnels, s'est évidemment trouvée à donner crédit à une conception sociologique des droits linguistiques au Canada ${ }^{52}$. Le problème tient toutefois au fait que la Cour s'est refusée, comme nous l'avons $\mathrm{vu}^{53}$, à donner tout contenu au principe de l'égalité des langues officielles, lequel avait pourtant fait l'objet d'un tel compromis politique et lequel s'était retrouvé consacré, pour les fins fédérales à tout le moins, au paragraphe 16(1) de la Charte de 1982. L'égalité des langues officielles au Canada ne repose donc sur aucune assise véritable et, de ce fait, ne jouit que d'un caractère théorique.

En ce qui nous concerne, nous estimons que l'égalité des langues officielles au Canada devrait pouvoir reposer, dans les faits, sur la consécration du concept des deux peuples fondateurs dans la Constitution du pays et sur l'égalité formelle de ceux-ci. Il nous semble en effet que le Canada est un pays bilingue parce que, précisément, il est constitué de deux sociétés. En ce sens pouvons-nous affirmer que les deux langues officielles seront réellement sur un pied d'égalité au Canada le jour où les deux nations constituant ce pays le seront elles-mêmes, à tout le moins sur le plan juridique.

52. Voir Société des Acadiens c. Association of Parents, précité, note 49, p. 578, où le juge Beetz a établi une distinction entre les droits linguistiques et les autres droits fondamentaux. Notons également que le juge en chef Dickson devait affirmer, dans Société des Acadiens, p. 566, que «les droits linguistiques revêtent un caractère fondamentalement et profondément social», alors que la juge Wilson devait reconnaître dans le même ordre d'idées, p. 639, que «les droits linguistiques constituent une réaction aux particularités historiques du Canada. Ces droits ont un contenu socio-culturel [...]».

53. Voir supra, note 49. 
Bien entendu, la reconnaissance constitutionnelle de la spécificité québécoise, qui était proposée dans l'Accord du lac Meech et dans l'Entente de Charlottetown, aurait constitué un pas dans la bonne direction, en ce qu'elle se serait trouvée à reconnaître à la fois le particularisme du Québec ${ }^{54}$, pierre d'assise des francophones d'Amérique du Nord, et le caractère bi-national du Canada. Une telle reconnaissance aurait toutefois été plus intéressante et plus prometteuse si, au lieu d'être purement symbolique comme c'était le cas, elle avait elle-même été porteuse de droits et de pouvoirs particuliers pour le peuple québécois. Il n'aurait s'agit là, en fait, que de la reconnaissance de droits nationaux, de droits sociologiques destinés essentiellement à profiter à la communauté française du Québec, un groupe majoritaire sur son territoire certes, mais néanmoins minoritaire dans l'ensemble canadien et fortement menacé en Amérique. Sans compter que le Québec est investi d'une mission particulière dans le contexte canadien, soit celle d'assurer la survie et l'affirmation de la langue française. Comme devait en effet l'affirmer M. Keith Spicer, alors qu'il était commissaire aux langues officielles du Canada :

«Ce n'est évidemment pas dans les communautés francophones des provinces à majorité anglophone, ni même dans les écoles de langues du gouvernement fédéral, que peuvent se déployer les efforts décisifs pour protéger et enrichir le patrimoine linguistique français au Canada. Ce qui se fait dans ces domaines est indispensable et digne d'un appui soutenu. Mais à longue échéance, l'avenir du français en Amérique du Nord dépendra surtout de la façon dont le Québec saura affermir sa principale langue de culture pour en faire une langue de travail et d'échanges sociaux. En définitive, la vitalité du français partout au Canada reposera sur le dynamisme, voire la saine

54. À titre d'exemple, mentionnons que la reconnaissance constitutionnelle du caractère distinct du Québec, qui était proposée dans l'Accord du lac Meech, aurait peut-être pu favoriser une interprétation de l'article 1 de la Charte de 1982 qui eut davantage tenu compte de l'identité particulière du Québec. En conséquence, l'article 1 en question aurait pu ultimement entraîner une application des droits et libertés de la Charte qui eut été différente, selon que cette application eut concerné le Québec ou une autre province canadienne.

Quant à l'Entente de Charlottetown, les chances que le principe de la société distincte eut pu influencer l'interprétation de l'article 1 de la Charte de 1982 étaient considérablement diminuées, du fait de l'obligation qui était faite au Québec (et aux autres provinces canadiennes) de favoriser l'épanouissement et le développement de sa communauté minoritaire de langue officielle. 
prédominance, du français dans ce seul territoire où les francophones forment la majorité et possèdent les institutions qui reflètent cette réalité» ${ }^{55}$.

Ces paroles de Keith Spicer illustrent le rôle particulier du Québec dans la réalisation du défi linguistique et culturel canadien; mais elles posent du même coup tout le problème de savoir autour de quels principes doivent s'organiser et se développer les relations entre la majorité française et la minorité anglaise du Québec. Ceci nous amène à examiner, dès à présent, la nature des rapports linguistiques ayant cours dans cette province.

\section{TROISIÈME PARTIE : $\quad$ LES RAPPORTS ENTRE LA MAJORITÉ FRANÇAISE ET LA MINORITÉ ANGLAISE DU QUÉBEC}

Il n'y a aucun doute dans notre esprit que la minorité anglaise du Québec a droit à ce que sa différence linguistique et culturelle soit reconnue dans la province, au même titre que la minorité française doit pouvoir jouir de cette même prérogative dans le contexte de l'ensemble du Canada. Ce droit découle directement, avons-nous vu, de la dualité nationale du pays et du principe de l'égalité des deux groupes fondateurs que celle-ci sous-tend.

Il n'en reste pas moins que les francophones sont majoritaires au Québec mais minoritaires dans l'ensemble du Canada, alors que c'est l'inverse pour les anglophones. Il n'est donc pas aisé d'identifier, dans le contexte québécois, quelle est la langue qui est réellement dominante et quelle est celle qui est réellement dominée.

En ce qui nous concerne, nous estimons que les rapports entre les francophones et les anglophones du Québec doivent être abordés sous l'angle plus général des relations qu'entretiennent les communautés française et anglaise dans l'ensemble canadien. En d'autres termes, nous croyons que le problème québécois en matière de langue ne doit pas être dissocié de l'ensemble du

55. K. SPICER, Commissaire aux langues officielles: premier rapport annuel, 1970-1971, Ottawa, Information Canada, 1971, p. 4. 
problème canadien, et que seul ce dernier est en mesure de donner au premier sa juste perspective.

Il ne faut pas oublier en effet que c'est la langue française qui est menacée en Amérique et, en conséquence, qu'il n'est que normal que le Québec prenne tous les moyens pour en assurer la survie. La Cour suprême du Canada a d'ailleurs elle-même reconnu, dans l'affaire $F_{o r d}{ }^{56}$, la vulnérabilité de la langue française au Canada et au Québec, l'importance et la légitimité de l'objet législatif de la Charte de la langue française ${ }^{57}$, ainsi que le fait que cette dernière était destinée à répondre à un besoin réel et urgent.

Inutile donc de mentionner que la vitalité du français au Canada ne saurait reposer que sur la saine prédominance de cette langue dans ce seul territoire où les francophones sont majoritaires: le Québec. Seule cette prédominance, en effet, est de nature à favoriser l'égalité des deux langues officielles du Canada, en rendant justement l'usage de celles-ci plus égal dans l'ensemble du pays. Il appert effectivement que l'égalité linguistique peut parfois impliquer la prédominance d'une langue sur une autre, surtout si cette prédominance sert à rendre les deux langues plus égales dans les faits.

Il ne saurait dès lors être question, au Québec, de mettre les langues anglaise et française sur un même pied d'égalité juridique. En effet, la langue française, bien que majoritaire au Québec, est beaucoup plus menacée que l'anglais dans le continent américain. L'égalité linguistique commande donc que le français bénéficie, au Québec, d'un certain avantage normatif par rapport à la langue minoritaire, l'anglais, cette dernière jouissant du précieux point d'appui que constitue l'ensemble de l'Amérique du Nord pour s'exprimer.

Notons d'ailleurs que l'idée voulant que le français jouisse d'un statut supérieur à l'anglais au Québec se concilie parfaitement avec la nature même des droits linguistiques, lesquels, n'étant pas des droits fondamentaux au sens

56. Voir: Ford c. P. G. (Québec), précité, note 51, pp. 777-778.

57. Charte de la langue française, L.R.Q., ch. C-11. Cette Charte est fréquemment désignée par le terme «Loi 101». C'est par ce terme que nous l'avons d'ailleurs désignée précédemment (voir supra, note 51), et que nous la désignerons ci-après. 
traditionnel ${ }^{58}$, ne sauraient être vus comme étant absolus et ne sauraient dans tous les cas être reconnus sur une base de stricte égalité. Voilà pourquoi il est fréquemment nécessaire de trouver, dans la législation en matière de langue, une solution qui réconcilie la prépondérance d'une langue sur une autre, tout en assurant la préservation et l'épanouissement de cette dernière.

On comprendra aisément que, dans le contexte québécois, tous ces principes posent la question de savoir comment rendre compatible la promotion du caractère distinct du Québec avec la vitalité et l'épanouissement de sa minorité anglophone ${ }^{59}$. Mais ces principes appellent également la question de savoir comment concilier, dans la législation du Québec, la prédominance du français avec la préservation des droits les plus légitimes de la minorité anglaise.

\section{La promotion du caractère distinct du Québec et l'essor de sa minorité anglaise}

L'Accord du lac Meech mettait en opposition la reconnaissance de la spécificité québécoise avec celle de la dualité canadienne. Quant à l'Entente de Charlottetown, elle reconnaissait à la fois le caractère distinct du Québec et l'attachement des gouvernements du pays à l'épanouissement et au développement des communautés minoritaires de langue officielle ${ }^{60}$.

D'éminents constitutionnalistes ont déjà émis l'opinion, dans le contexte du débat ayant entouré l'Entente de Charlottetown, qu'entre le principe de la spécificité québécoise et celui de l'épanouissement de la minorité anglaise du Québec, la Cour suprême du Canada en serait vraisemblablement venue à privilégier ce dernier. Selon ces juristes, en effet, la préférence de la Cour suprême pour le «principe de l'épanouissement» aurait essentiellement été fondée sur le penchant naturel qu'elle a toujours manifesté en faveur de la protection des intérêts minoritaires plutôt que majoritaires. Plus particulièrement, comme devaient l'affirmer les constitutionnalistes en question:

58. Voir supra, note 52.

59. Cette question avait en effet été posée avec acuité par l'Entente de Charlottetown de 1992: voir supra, note 21 .

60. Ce principe sera ci-après désigné par le terme «principe de l'épanouissement». 


\begin{abstract}
«[N]ous sommes d'avis que la clause de la société distincte du Projet d'Accord constitutionnel n'a virtuellement aucune valeur pour le Québec. Nous considérons même que son adoption marquerait un recul par rapport à la situation actuelle, car dans son jugement sur la langue de la publicité et de l'affichage commercial, la Cour suprême du Canada a déjà reconnu que la Constitution peut, dans une certaine mesure, être interprétée en tenant compte du caractère distinct du Québec. Or, le Projet d'Accord constitutionnel, en réduisant la société distincte à certaines réalités et en la plaçant sur le même pied que l'engagement des gouvernements envers l'épanouissement et le développement de la minorité anglophone du Québec, pourrait remettre en question la portée de cet acquis» ${ }^{61}$.
\end{abstract}

En ce qui nous concerne, nous partageons entièrement ce point de vue. Nous estimons en effet que le «principe de l'épanouissement» aurait été appelé, s'il avait effectivement été constitutionnalisé, à faire l'objet d'une interprétation toujours de plus en plus libérale de la part de la Cour suprême du Canada. C'est ainsi que le «principe de l'épanouissement» aurait pu en venir éventuellement à annihiler les effets du principe de la société distincte, voire à marquer un véritable recul pour la compétence du Québec en matière de langue. Plus particulièrement, nous considérons que l'alinéa 2b) de la Charte de 1982, reconnaissant notamment la liberté d'expression, se serait révélé être une véritable boîte de Pandore lorsqu'il aurait dû être interprété à la lumière du «principe de l'épanouissement» de l'Entente de Charlottetown.

L'idéal serait donc, à notre avis, que l'on revienne essentiellement à la formulation de l'Accord du lac Meech, tout en la modifiant quelque peu. L'Accord du lac Meech, en effet, nous semblait répondre adéquatement à la double préoccupation de promouvoir le caractère distinct du Québec et de protéger la dualité canadienne. Nous aurions toutefois souhaité que, contrairement à ce que proposait l'Accord du lac Meech, le principe du caractère distinct du Québec soit tout autant reconnu comme étant une caractéristique

61. H. BRUN et autres, «La clause relative à la société distincte du Rapport du consensus sur la Constitution: un recul pour le Québec», dans C. BARITEAU et autres, Référendum, 26 octobre 1992: Les objections de 20 spécialistes aux offres fédérales, Montréal, Éditions Saint-Martin, 1992, p. 56. Notons que ce texte a aussi été publié dans le journal Le Devoir du 4 septembre 1992, sous le titre «La "société distincte" ne veut plus rien dire». 
fondamentale du pays que l'était celui de la dualité canadienne. Nous aurions également souhaité que la reconnaissance de la spécificité du Québec s'accompagne d'une dévolution particulière de pouvoirs législatifs et de l'octroi d'un veto constitutionnel en sa faveur, ainsi que d'une consécration formelle du concept des deux peuples fondateurs dans la Constitution du Canada.

Une chose est certaine toutefois, et c'est que l'épanouissement de la minorité anglaise du Québec devrait continuer, dans l'hypothèse d'une révision de la Constitution du pays, à être laissé au soin des autorités québécoises. Cette suggestion nous amème évidemment à constater jusqu'à quel point il est impérieux que le Québec se sensibilise lui-même à l'idée d'assurer l'essor de sa minorité anglophone par le biais de sa propre législation, tout en ayant pour priorité d'assurer la survie et le rayonnement de la langue française au Québec et au Canada.

\section{La législation québécoise en matière de langue}

Il ne fait aucun doute que les rapports entre la majorité française et la minorité anglaise du Québec doivent être marqués du respect des droits et privilèges de cette dernière, tout projet d'assimilation étant non seulement indéfendable mais au surplus carrément irréalisable. Il va sans dire également que la majorité francophone du Québec ne doit pas chercher obstinément et inutilement à refuser à sa minorité anglophone les droits spécifiques qu'ellemême ne voudrait pas qu'on lui conteste.

En fait, nous estimons que le Québec a le devoir moral de procéder à un aménagement linguistique qui réponde aux besoins essentiels de sa minorité anglophone, tout en ne remettant pas en cause la cohésion même de la nation québécoise, dont l'une des caractéristiques est précisément d'être le bastion de la francophonie en Amérique. En d'autres termes, le Québec doit chercher à établir un certain compromis entre les droits linguistiques de sa majorité et de sa minorité, tout en respectant les exigences qu'imposent la sauvegarde et la cohérence $^{62}$ de la nation qu'il constitue.

62. Le concept de «cohésion nationale» ne vise pas l'exclusion des anglophones du Québec dans la définition de la nation québécoise, mais bien plutôt leur participation active à une société où le français sera reconnu comme étant la langue officielle de la vie publique nationale. 
Que l'on nous comprenne bien toutefois! L'idée n'est pas d'encourager le pluralisme linguistique au Québec, ni même le bilinguisme dans toutes les affaires provinciales. Au contraire, il s'agit d'encourager la primauté du français dans tous les secteurs de la vie sociale où cette langue est la plus menacée (ex. les relations de travail), voire l'unilinguisme officiel dans tel ou tel domaine. Assurer la prépondérance ${ }^{63}$ du français au Québec nous semble en effet être un objectif d'autant plus légitime que celui-ci n'a jamais empêché l'octroi de privilèges et de services importants à la minorité anglophone de la province, sans doute la plus choyée au Canada.

Dans cette veine, le Québec s'était donné par la Loi 101 et par ses politiques culturelles, la mission de favoriser l'émergence d'un peuple pluriethnique dont la langue publique commune devait être le français. En effet, la Loi 101 était à l'origine motivée par le désir de faire du français la langue de l'État québécois et de la Loi, aussi bien que la langue normale et habituelle du travail, de l'enseignement, des communications, du commerce et des affaires. La Loi 101 cherchait toutefois à s'inscrire dans le respect des institutions de la communauté d'expression anglaise et dans le respect des autres groupes minoritaires de la province, dont elle reconnaissait d'ailleurs d'emblée l'apport précieux au développement du Québec.

La Loi 101 a cependant été sérieusement affectée par un certain nombre de décisions de la Cour suprême du Canada intervenues depuis son adoption. Ces décisions étaient motivées par l'article 133 de la Loi de $1867^{64}$, par l'article 23 de la Charte de $1982^{65}$, ou par la liberté d'expression reconnue à l'alinéa 2b)

63. La prépondérance de la langue française peut évidemment être assurée par une certaine primauté ou prédominance par rapport à la langue anglaise, voire par l'usage exclusif du français lorsque cela est opportun.

64. Voir: Procureur général du Québec c. Blaikie, précité, note 36, où la Cour suprême du Canada a déclaré inopérant le chapitre III (art. 7 à 13 portant sur la langue de la législation et de la justice) de la Loi 101, puisqu'il contrevenait à l'article 133 de la Loi de 1867.

65. Voir: P.G. Québec c. Quebec Protestant School Boards, [1984] 2 R.C.S. 66, où la Cour suprême du Canada a déclaré inopérants les articles 72 et 73 de la Loi 101 (sur la langue de l'enseignement) en raison de leur incompatibilité avec l'article 23 de la Charte de 1982. 
de la même Charte ${ }^{66}$. C'est ainsi que, le 17 juin 1993, l'Assemblée nationale du Québec devait adopter des mesures visant à harmoniser certaines des dispositions de la Loi 101 avec les décisions judiciaires en question ${ }^{67}$.

En réalité, la Loi modifiant la Charte de la langue française démontre éloquemment l'ampleur des contraintes constitutionnelles qui s'imposent actuellement au Québec dans le domaine linguistique. Ces contraintes empêchent le Québec de se doter des outils dont il estime avoir besoin pour assurer la promotion de la langue française sur son territoire.

Le Québec est donc aux prises, en matière de langue, avec un véritable «carcan constitutionnel», l'empêchant d'agir et d'accomplir sa propre destinée comme il l'entend. Pourtant, nous sommes d'avis que l'enjeu que représente la survie de la langue française en Amérique serait de nature à justifier amplement que le Québec affirme et précise nettement ses propres règles concernant le statut et l'usage du français pour les fins provinciales, et s'assure du respect de celles-ci. C'est dans cette optique, d'ailleurs, que nous estimons que le Québec

66. Voir: Ford c. P. G. (Québec), précité, note 51, où la Cour a déclaré inopérant l'article 69 de la Loi 101 (sur la langue des raisons sociales), puisqu'il était contraire à l'alinéa 2b) (liberté d'expression) de la Charte de 1982. Le même raisonnement pouvait également être appliqué à l'égard de l'article 58 de la Loi 101, en matière de langue de l'affichage public et de la publicité commerciale.

67. Il s'agit de la Loi modifiant la Charte de la langue française, L.Q. 1993, ch. 40.

Comme l'affirment les notes explicatives accompagnant cette loi modificatrice, celle-ci vise à harmoniser certaines des dispositions de la Loi 101 relatives à la langue de la législation et de la justice, à la langue du commerce et des affaires et à la langue de l'enseignement avec les décisions de la Cour suprême du Canada dont nous avons déjà parlé: voir supra, notes 64, 65 et 66 .

Évidemment, l'une des mesures les plus importantes de la Loi de 1993 en cause consiste à avoir permis l'usage d'une langue autre que le français dans l'affichage public et la publicité commerciale, «pourvu que le français y figure de façon nettement prédominante»: voir l'art. 18 de la Loi modificatrice, remplaçant les articles 58 à 58.2 de la Loi 101 par un nouvel article 58. «Notons cependant que ce nouvel article contient notamment les dispositions suivantes : Toutefois, le gouvernement peut déterminer, par règlement, les lieux, les cas, les conditions ou les circonstances où l'affichage public et la publicité commerciale doivent se faire sans prédominance du français ou uniquement dans une autre langue.»

Voir également, à titre de mesures particulièrement importantes, l'article 22 de la Loi de 1993, modifiant les dispositions de la Loi 101 en matière de raisons sociales, ainsi que les articles 23 à 35 de la Loi de 1993, modifiant la Loi 101 en matière de langue de l'éducation. 
pourrait légitimement -- en tenant compte des besoins ressentis et du contexte social -- poursuivre les objectifs suivants:

1. Chercher à affirmer l'existence et à promouvoir le développement d'une seule langue officielle, le français, la langue nationale du Québec.

Il s'agit essentiellement ici de reconnaître que la langue française constitue un élément essentiel de l'identité nationale du Québec, que cette langue appartient au patrimoine québécois et qu'elle se doit d'être la langue officielle de la vie publique nationale au Québec. Comme devait en effet l'affirmer le préambule de la Loi 101:

Langue distinctive d'un peuple majoritairement francophone, la langue française permet au peuple québécois d'exprimer son identité.

La législation québécoise en matière de langue ne doit donc pas être envisagée comme étant une simple composante du bilinguisme canadien, ni comme en étant le prolongement dans les matières relevant de la compétence du Québec, mais bien plutôt comme le plus précieux outil dont disposent les francophones en Amérique pour exprimer ce qu'ils sont, pour faire valoir leur identité nationale.

2. Adopter et mettre en oeuvre des politiques d'intégration à la langue et à la culture françaises, destinées particulièrement aux nouveaux arrivants.

\section{Chercher à consacrer le français comme étant le véhicule privilégié des relations économiques.}

Plus particulièrement, il s'agirait de reconnaître le droit à l'information commerciale en français. Ainsi, le français serait reconnu comme étant la langue obligatoire de la publicité commerciale imprimée. Une traduction dans une autre langue serait néanmoins permise, mais à la condition que le français soit aussi lisible et intelligible que celle-ci. 
Le français serait également consacré comme étant la langue exclusive de l'affichage commercial, à tout le moins à l'extérieur des commerces, et des raisons sociales. Il nous semble en effet que ces deux volets de la vie sociale québécoise sont étroitement liés à la capacité du Québec d'intégrer les nouveaux arrivants à la langue et à la culture françaises, voire à sa capacité d'assurer la cohésion de la nation québécoise et de donner à la province un visage français.

\section{Confirmer l'enseignement du français comme étant l'une des missions fondamentales de l'État québécois.}

\section{Renforcer le rôle de la langue française en matière de développements scientifiques et technologiques.}

\section{Renforcer le rôle de la langue française en matière de présentations de programmes de radio ou de télévision. \\ 7. Renforcer le droit de travailler en français.}

La poursuite des objectifs susmentionnés requiert évidemment que le Québec ne cesse de lutter pour la préservation, voire l'accroissement ou l'affirmation de son autonomie dans des secteurs clés pour la vie sociale, comme par exemple la langue, la culture, l'éducation, les affaires sociales, l'immigration, la radiodiffusion et les relations de travail.

Mais ce dernier point soulève en lui-même toute la question de savoir comment concilier les intérêts de la majorité francophone du Québec avec ceux des minorités francophones des autres provinces canadiennes. Il appert en effet qu'alors que la majorité québécoise recherche avidement l'accroissement de ses pouvoirs législatifs, les minorités francophones du pays espèrent pour leur part l'adoption de normes constitutionnelles protectrices à l'encontre de l'autonomie culturelle et linguistique des provinces à majorité anglophone.

On comprend aisément, dès lors, jusqu'à quel point il est impérieux que ce dilemme, qui a pour effet de diviser la communauté francophone de tout le pays, soit résolu et que le Québec saisisse l'occasion pour redéfinir ses liens avec les francophones du «reste du Canada». 


\section{QUATRIÈME PARTIE : L ES R A P P O R T S E N T R E L E S FRANCOPHONES DU QUÉBEC ET CEUX DES A U T RES PROVINCES CANADIENNES}

Le Québec n'a pas toujours cherché à établir des ponts avec les francophones qui vivent et s'expriment à l'extérieur de son territoire. On constate même que, depuis un certain nombre d'années, les liens entre ces deux communautés sont de moins en moins nourris. Il faut bien admettre cependant que les intérêts de chacune d'elles sont souvent en complète opposition, comme devait d'ailleurs le souligner le professeur José Woehrling ${ }^{68}$. Par ailleurs, on constate que les francophones «hors Québec», comme on se plaît souvent à les appeler, délaissent de plus en plus le concept de «Canada français», auquel ils étaient pourtant historiquement associés, et cherchent à définir d'une nouvelle façon leur appartenance culturelle et linguistique à la vie française en Amérique.

Pourtant, les communautés francophones qui se retrouvent en situation minoritaire au Canada ont besoin d'un Québec fort et respecté, puisque l'essor de la francophonie au Canada dépend essentiellement du Québec et de sa vitalité. En effet, il appert que, par suite de la répartition territoriale des francophones au Canada, seul le Québec, «une province pas comme les autres», peut accomplir cette délicate mission que constitue le fait d'assurer la survivance de la francophonie en Amérique.

Mais le Québec aussi a besoin des minorités francophones du Canada, s'il ne veut pas devenir un ghetto. On constate cependant que, avec le temps, le gouvernement du Québec s'est coupé des communautés francophones minoritaires (les Acadiens, les Franco-Ontariens, les Franco-Manitobains, etc.), en se faisant le défenseur d'un nationalisme trop souvent exclusif et insensible aux préoccupations de ces dernières.

68. Voir J. WOEHRLING, La Constitution canadienne et les droits linguistiques: convergences et divergences entre les intérêts des Québécois francophones, de la minorité angloquébécoise et des minorités francophones du Canada, Communication présentée dans le cadre du Colloque intitulé Les droits linguistiques au Canada: collusions ou collisions?, tenu par le Centre canadien des droits linguistiques à Ottawa, du 4 au 6 novembre 1993, pp. 53-55. 
Lorsque le nationalisme québécois se donne comme mandat de réaliser la pleine souveraineté juridique et politique du Québec, l'insouciance ou l'indifférence à l'égard du sort des minorités de langue française du Canada peut se comprendre, à la limite ${ }^{69}$. Mais lorsque le nationalisme québécois repose sur le désir d'assurer au Québec un statut particulier au sein du fédéralisme canadien, voire de lui donner un statut d'égalité avec le Canada anglais, alors l'exclusion des francophones minoritaires au Canada nous paraît être une grave erreur.

Il nous semble en effet que la dualité canadienne, ainsi que le concept des deux peuples fondateurs sur lequel cette dualité repose, commandent que les rapports linguistiques et culturels au Canada soient envisagés sous un angle pancanadien, plutôt que sous un angle strictement québécois ${ }^{70}$. C'est-à-dire que la dualité devrait être abordée dans une perspective «Canada françaislCanada anglais», plutôt que simplement «Québec français\Canada anglais». De cette façon, certains anglophones du Canada seront un peu moins tentés de définir le bilinguisme comme signifiant un Québec français (avec d'importants privilèges pour sa minorité) et le «reste du Canada», anglais.

$\mathrm{Au}$ fond, peut-être la meilleure façon de concilier les intérêts de la majorité québécoise avec ceux des minorités francophones des autres provinces autant acceptable, ni qu'elle soit généralisée parmi les sécessionnistes québécois. À cet égard, il peut être intéressant de noter que le Parti Québécois se donne actuellement pour politique, advenant la sécession du Québec, de «veiller à aménager un cadre de coopération active entre le Québec et les minorités francophones du Canada [...]»: voir Le Québec dans un monde nouveau (Conseil exécutif national du Parti Québécois), Montréal, V.L.B. Éditeur et le Parti Québécois, 1993, p. 77. C'est ainsi que le Québec pourrait songer, advenant son accession à la pleine souveraineté étatique, à négocier avec les gouvernements du Canada anglais des accords de réciprocité en vertu desquels les différents signataires s'engageraient à reconnaître des droits et privilèges comparables aux minorités de langue officielle placées sous leurs juridictions respectives: voir J. WOEHRLING, «Les aspects juridiques de la redéfinition du statut politique et constitutionnel du Québec», dans Éléments d'analyse institutionnelle, juridique et démolinguistique pertinents à la révision du statut politique et constitutionnel du Québec, Québec, Commission sur l'avenir politique et constitutionnel du Québec, document de travail no 2, 1991, p. 63. Il y a lieu de prendre connaissance également des mesures qui figurent dans le Programme du Parti Québécois intitulé Des idées pour mon pays, Montréal, le Parti Québécois, 1994, pp. 25 et 28.

70. Sur cette question, il y a lieu de voir: J. WOEHRLING, loc. cit., note 68, pp. 49-52. 
canadiennes consisterait-elle, comme devait le suggérer le professeur José Woehrling, ...

«... à introduire dans les dispositions constitutionnelles susceptibles d'avoir un effet sur les droits linguistiques un élément d'asymétrie qui permettrait d'en limiter les effets en ce qui le concerne [le Québec], tout en autorisant leur pleine application dans le reste du Canada» ${ }^{71}$.

Souhaitons que la situation ci-haut décrite se réalise un jour, tout en demeurant toutefois résolument réalistes quant aux minces chances qu'il en soit ainsi.

Mais souhaitons également que les liens entre les francophones du Québec et ceux des autres provinces canadiennes soient redéfinis à la lumière d'un nouveau concept à la fois moderne et englobant. Après tout, les francophones de tout le Canada, majoritaires ou minoritaires, n'ont-ils pas en commun cette volonté de survivre malgré les forces et les pressions d'un environnement qui leur est parfois hostile, souvent indifférent?

\section{CONCLUSION}

Nous avons tenté, dans le présent texte, de jeter un éclairage sur les rapports fondamentaux qui existent entre les communautés francophones et anglophones du Canada.

C'est ainsi que nous avons souligné jusqu'à quel point cette réalité historique et sociologique qu'est le caractère distinct du Québec expliquait l'ensemble des relations linguistiques et culturelles au pays, et impliquait une définition du Canada en tant qu'État «bi-national». Nous avons affirmé par ailleurs que le Québec, en tant qu'assise de la francophonie en Amérique, avait des responsabilités particulières, des devoirs que n'avaient pas les autres provinces canadiennes. Nous avons vu enfin que la personnalité distincte du Québec était intimement liée à son droit à l'autodétermination politique sur la scène internationale.

71. Id., p. 55 . 
En fait, nous croyons que l'égalité des deux grandes communautés linguistiques composant ce pays devrait être consacrée en tant que pilier du fédéralisme canadien. Dans le contexte québécois cependant, cette recherche de l'égalité pourrait entraîner la reconnaissance législative de la prééminence de la langue française sur la langue anglaise, voire de son usage exclusif dans certains secteurs de la vie sociale. La langue française est en effet fortement menacée en Amérique et a besoin de cette même reconnaissance pour que s'installe une plus grande égalité, dans les faits, entre les deux langues officielles au Canada.

Bien entendu, c'est le vieux rêve dualiste, que tant de Québécois ont caressé au fil des ans, qui permet d'envisager un tant soit peu l'égalité des deux communautés de langue officielle au Canada. Pourtant, ce rêve s'est brisé, quelque part après 1982, contre les récifs d'un fédéralisme canadien devenu inflexible et insensible aux aspirations du Québec. Comme devait en effet l'affirmer le professeur Guy Laforest:

«Ce qui rend suranné notre rêve canadien, et impossible la dualité, c'est le désir, dans la logique du système fédéral canadien, de broyer la dimension collective de l'identité des Québécois» ${ }^{72}$.

L'heure des choix pourrait bien sonner à nouveau pour le Québec, puisqu'il est possible que l'option sécessionniste soit soumise à l'examen des Québécois à l'occasion d'un référendum prévu pour 1995. Si tel devait être le cas, il est plausible de penser que les forces fédéralistes tenteront d'offrir une alternative aux Québécois en relançant la question de l'aménagement de l'Union canadienne, voire en rouvrant le dossier de la réforme constitutionnelle au Canada. Quoi qu'il en soit, le Québec se trouvera vraisemblablement placé, une fois de plus, devant son «destin national», et devra s'autodéterminer en fonction de ce qu'il jugera être le plus approprié pour son avenir.

Souhaitons simplement que le Québec ait la sagesse, quel que soit le choix qu'il s'apprête à poser, de chercher à édifier les bases d'une cohabitation harmonieuse avec sa minorité anglophone. Celle-ci, en effet, doit continuer de 
faire partie intégrante de la société québécoise. Par ailleurs, non seulement les droits individuels des membres de la minorité anglophone doivent-ils être reconnus dans la mesure la plus large possible, mais en plus celle-ci doit-elle pouvoir continuer de compter sur un ensemble d'institutions scolaires, sociales et culturelles propres à assurer son dynamisme.

Mais souhaitons également que le Québec ait la sagesse de chercher à renouer des liens féconds avec la minorité française des autres provinces canadiennes. Les francophones de tout le pays, en effet, sont appelés à participer au même projet collectif: assurer la survie et l'épanouissement du français au Canada. Cette mission, pour exaltante qu'elle soit, ne pourra toutefois s'accomplir qu'à la condition d'être soutenue par un vaste réseau de solidarité. Ceci implique notamment que le nationalisme québécois soit ouvert sur l'extérieur et que le Québec soit en étroite relation avec ses partenaires privilégiés en Amérique: les francophones vivant en situation minoritaire. 